Boise State University

ScholarWorks

Electrical and Computer Engineering Faculty

Department of Electrical and Computer

Publications and Presentations

Engineering

9-1-2007

Finite-Difference Time Domain Method for Nonorthogonal Unit-Cell Two-Dimensional Photonic Crystals

Wan Kuang

Boise State University

Woo J. Kim

Samsung Electronics

John D. O'Brien

University of Southern California, Los Angeles

This document was originally published by IEEE in Journal of Lightwave Technology. Copyright restrictions may apply.

DOI: 10.1109/JLT.2007.903827 


\title{
Finite-Difference Time Domain Method for Nonorthogonal Unit-Cell Two-Dimensional Photonic Crystals
}

\author{
Wan Kuang, Member, IEEE, Woo Jun Kim, and John D. O'Brien, Senior Member, IEEE
}

\begin{abstract}
A finite-difference time-domain (FDTD) method based on a regular Cartesian Yee's lattice is developed for calculating the dispersion band diagram of a 2-D photonic crystal. Unlike methods that require auxiliary difference equations or nonorthogonal grid schemes, our method uses the standard centraldifference equations and can be easily implemented in a parallel computing environment. The application of the periodic boundary condition on an angled boundary involves a split-field formulation of Maxwell's equations. We show that the method can be applied for photonic crystals of both orthogonal and nonorthogonal unit cells. Complete and accurate bandgap information is obtained by using this FDTD approach. Numerical results for 2-D TE/TM modes in triangular lattice photonic crystals are in excellent agreement with the results from 2-D plane wave expansion method. For a triangular lattice photonic crystal slab, the dispersion relation is calculated by a 3-D FDTD method similarly formulated. The result agrees well with the 3-D finite-element method solution. The calculations also show that the 2-D simulation using an effective index approximation can result in considerable error for higher bands.
\end{abstract}

Index Terms-Band diagram, finite-difference time domain (FDTD), photonic crystals, triangular lattice.

\section{NOMENCLATURE}

FDTD Finite-difference time domain.

PWE Plane wave expansion.

FEM Finite-element method.

PML Perfectly matched layer.

ABC Absorbing boundary condition.

\section{INTRODUCTION}

$\mathbf{T}$ HE periodic spatial modulation of the refractive index in photonic crystals has proven to be an effective way to modify the flow of light [1]-[3]. Strong diffraction and multiple scattering in periodic structures generally require Maxwell's

Manuscript received January 15, 2007; revised July 2, 2007. This work was supported by the National Science Foundation under Grants 0321233 and ECS 0094020. The work of W. Kuang was supported by Idaho Space Consortium and NASA EPSCoR.

W. Kuang is with the Boise State University, Boise, ID 83725 USA (e-mail: wankuang@boisestate.edu).

W. J. Kim is with Samsung Electronics, Seoul, 100-742, South Korea.

J. D. O'Brien is with the University of Southern California, Los Angeles, CA 90089 USA, and also with the University of South Carolina, Columbia, SC 29208 USA (e-mail: jdobrien@usc.edu).

Color versions of one or more of the figures in this paper are available online at http://ieeexplore.ieee.org.

Digital Object Identifier 10.1109/JLT.2007.903827 equations to be numerically solved. FDTD method has been widely used in modeling photonic crystal devices. It linearly scales with the simulation dimension and can be implemented in a parallel computing environment very efficiently.

However, the application of the FDTD method in a band structure calculation has been limited to photonic crystals with orthogonal unit cells. This is mainly because the general second-order accurate FDTD method in the Cartesian coordinate presents a discrete approximation for fields based on a uniform orthogonal grid. Except for the photonic crystals in a rectangular lattice, the physical boundary of the unit cell does not directly conform to the FDTD grid. The desired periodic boundary condition cannot be directly enforced on the physical boundary but rather on an auxiliary boundary, which is a staircased approximation of the physical boundary. Unfortunately, this leads to instability, regardless of how fine the mesh is made to resolve the boundary contour, as the electromagnetic field at the boundary carries important phase information.

Several matrix-based methods, for example, PWE and FEM, have been used for calculating the band structures of photonic crystals. For a full 3-D simulation of a 2-D photonic crystal slab, a supercell approach is commonly employed. Since the calculation burden quadratically scales with the simulation dimension, most of the calculations have been limited to two dimensions using an effective index approximation. Twodimensional photonic bandgap calculations agree well with full 3-D calculations; however, the calculated group velocity considerably deviates from the 3-D simulations. Three-dimensional PWE and FEM are computationally intensive. Although the amount of computation in a 3-D simulation can be reduced by invoking additional symmetry considerations [4], in many cases, such procedures may not be enough. For instance, simulation of the surface plasmon polaritons in metal disk arrays requires the electromagnetic field to be sampled at a $1-\mathrm{nm}$ distance or smaller [5]. Parallelization for these matrix-based methods has been more difficult and less efficient than for the FDTD [6]. As parallel computing facilities become increasingly available, it would be desirable to utilize the strength of the FDTD in parallelization for band structure calculation.

In this paper, an FDTD method is presented for calculating the dispersion relation of general 2-D photonic crystals. Unlike methods that require auxiliary difference equations [7] or nonorthogonal grid schemes [8], our method uses the standard central-difference equations in an orthogonal grid scheme and can be easily implemented in a parallel computing environment. 
The band diagram of a triangular lattice photonic crystal slab is calculated by a 2-D FDTD using an effective index approximation and a full 3-D FDTD simulation. The results agree extremely well with those given by the 2-D PWE and 3-D FEM approaches, respectively.

\section{METHOD AND IMPLEMENTATION}

The FDTD method, which was introduced by Yee in 1966, is a marching-in-time procedure that simulates continuous actual electromagnetic waves by volumetric sampling of unknown fields [9], [10]. For photonic crystals with a spatially periodic dielectric constant $\varepsilon(\boldsymbol{r}+\boldsymbol{R})=\varepsilon(\boldsymbol{r})$, where $\boldsymbol{R}$ is the primitive lattice vector, the electromagnetic fields $\boldsymbol{E}(\boldsymbol{r})$ and $\boldsymbol{H}(\boldsymbol{r})$ are characterized by a wave vector $\boldsymbol{k}$ in the reduced Brillouin zone and band index $n$ according to Bloch's theorem

$$
\begin{aligned}
\boldsymbol{E}(\boldsymbol{r}) & =\boldsymbol{E}_{n, \boldsymbol{k}}(\boldsymbol{r})=\boldsymbol{u}_{n, \boldsymbol{k}}(\boldsymbol{r}) \exp (i \boldsymbol{k} \cdot \boldsymbol{r}) \\
\boldsymbol{H}(\boldsymbol{r}) & =\boldsymbol{H}_{n, \boldsymbol{k}}(\boldsymbol{r})=\boldsymbol{v}_{n, \boldsymbol{k}}(\boldsymbol{r}) \exp (i \boldsymbol{k} \cdot \boldsymbol{r})
\end{aligned}
$$

in which $\boldsymbol{u}_{n, \boldsymbol{k}}(\boldsymbol{r})$ and $\boldsymbol{v}_{n, \boldsymbol{k}}(\boldsymbol{r})$ are periodic functions that satisfy

$$
\begin{aligned}
& \boldsymbol{u}_{n, \boldsymbol{k}}(\boldsymbol{r}+\boldsymbol{R})=\boldsymbol{u}_{n, \boldsymbol{k}}(\boldsymbol{r}) \\
& \boldsymbol{v}_{n, \boldsymbol{k}}(\boldsymbol{r}+\boldsymbol{R})=\boldsymbol{v}_{n, \boldsymbol{k}}(\boldsymbol{r}) .
\end{aligned}
$$

From (3) and (4), it shows that the eigenvalue solutions in a periodic structure can be found in the unit cell for a given periodic boundary condition, as shown in (1) and (2). Because of the structural complexity, it is often solved with a variety of numerical methods that include the PWE [3], [11]-[14], the FEM [6], and the FDTD method. Reviews of those numerical methods and their pros and cons can be found in [6].

For the FDTD, a simulation starts from a random initial condition to not exclude any possible modes and propagates for an adequate amount of time to give sufficient precision. The eigenmodes are then given by the peaks of the Fourier transform of the complex field components in the time domain recorded at various low-symmetry locations for a given propagation constant. The choice of initial condition and the locations of probes are very important. An initial condition that is not random enough will not be able to excite all possible modes in the structure. Probes at the high-symmetry locations of the structure are incapable of detecting all eigensolutions, as some modes are likely to have nodes at those locations.

This approach is different from the PWE, FEM, and other frequency domain methods where the eigenvalues are directly solved. In the following, we will describe the FDTD algorithm for a general nonorthogonal unit cell. Without losing generality, it will be explained for a triangular lattice.

\section{A. Unit Cell}

Fig. 1 shows the real and reciprocal spaces in the $x-y$ plane of a triangular lattice photonic crystal. Assuming a lattice constant of $a$, the primitive vectors $\boldsymbol{a}_{1}$ and $\boldsymbol{a}_{2}$ are given by $a \hat{x}$ and $[(a / 2) \hat{x}]+[(\sqrt{3} a / 2) \hat{y}]$, respectively. The correspond-

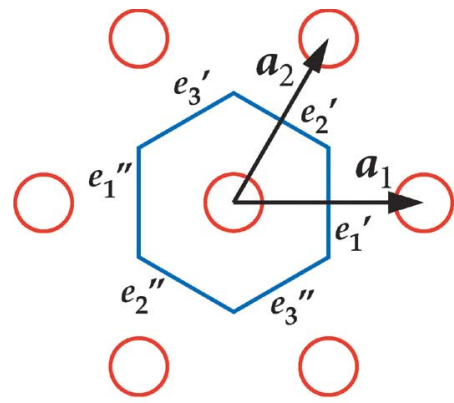

(a)

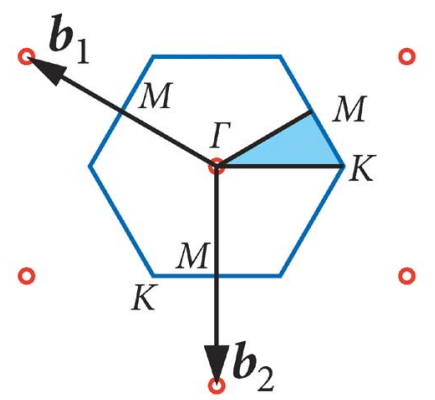

(b)

Fig. 1. (a) Real space and (b) reciprocal space representation of a triangular lattice where $\boldsymbol{a}_{1}, \boldsymbol{a}_{2}$ and $\boldsymbol{b}_{1}, \boldsymbol{b}_{2}$ are the unit vectors for the real space and reciprocal space, respectively.

ing reciprocal lattice vectors $\boldsymbol{b}_{1}$ and $\boldsymbol{b}_{2}$ are $\left[-(2 \pi / a) \hat{k_{x}}\right]+$ $\left[(2 \pi / \sqrt{3} a) \hat{k_{y}}\right]$ and $-(4 \pi / \sqrt{3} a) \hat{k_{y}}$, respectively. The highlighted triangle in Fig. 1(b) indicates the reduced Brillouin zone, where high-symmetry locations and their corresponding coordinates $\Gamma(0,0), M(\pi / a, \pi / a \sqrt{3})$, and $K(4 \pi / 3 a, 0)$ are labeled. For $M$ and $K$ points, other choices of coordinates exist, as shown in the figure.

Although the hexagonal Wigner-Seitz unit cell, as shown in Fig. 1(b), is generally explained in the literature as an example of a unit cell for the triangular lattice, it is, nevertheless, difficult to numerically handle. Among the three pairs of parallel periodic boundaries that are labeled as $e_{1}^{\prime}-e_{1}^{\prime \prime}, e_{2}^{\prime}-e_{2}^{\prime \prime}$, and $e_{3}^{\prime}-$ $e_{3}^{\prime \prime}$ in Fig. 1(a), only two of them are completely independent. The redundant Bloch equations lead to numerical instability. Additionally, the nonorthogonal unit cell does not natively conform to the Cartesian FDTD space grid. The periodic boundary condition cannot be directly enforced on the physical boundary.

We will show that a nonorthogonal FDTD grid scheme is unnecessary if the parallelogram unit cell, as shown in Fig. 2, is chosen instead. In this case, exactly two sets of periodic boundary conditions are enforced at the edges, i.e., $f_{1}^{\prime}-f_{1}^{\prime \prime}$ and $f_{2}^{\prime}-$ $f_{2}^{\prime \prime}$, of the unit cell. The electromagnetic field is periodically wrapped with appropriate phase conditions given by $\exp (-i \boldsymbol{k}$. $\boldsymbol{r})$, where $\boldsymbol{k}$ is the propagation constant of interest, and $\boldsymbol{r}$ is any of the primitive lattice vectors $\boldsymbol{a}_{1}$ or $\boldsymbol{a}_{2}$, depending on the associated periodic boundary. Taking the $\Gamma-M$ direction, for example, the propagation constant $\boldsymbol{k}$ is given by $(-2 \pi \xi / a \sqrt{3}) \hat{y}$, as shown in Fig. 1(b), in which $\xi \in[0,1]$ is a fraction; therefore, $k$ covers the whole range of the $\Gamma-M$ vector. The calculation requires a phase delay or advance of $\exp (-2 \pi \xi i)$ 


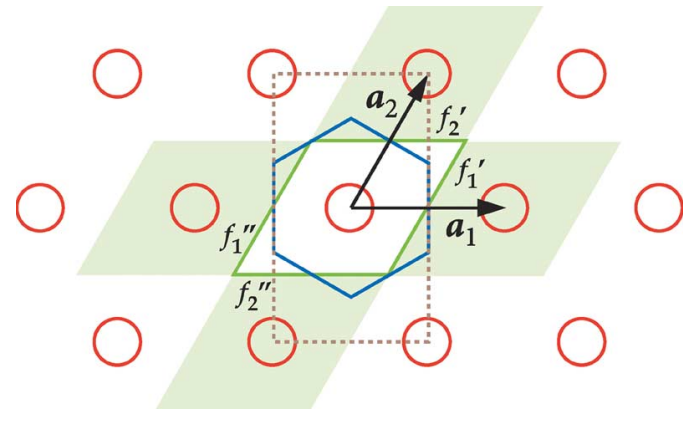

Fig. 2. Wigner-Seitz unit cell for a triangular lattice in the real space shown in blue lines and its equivalent rhombus unit cell in solid green lines employed in the numerical calculations. The light-green-shaded area indicates the periodicity of the lattice by applying rhombus unit cell.

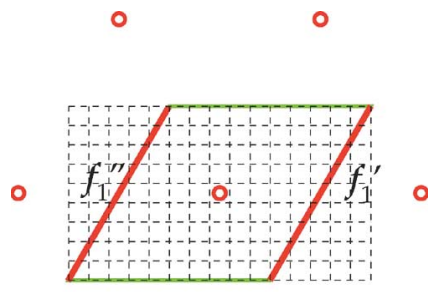

(a)

o

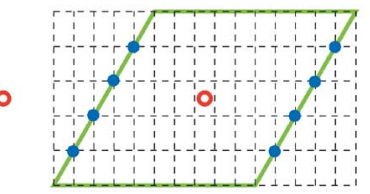

(b)
Fig. 3. (a) Uniform Cartesian grids that cannot completely sample the boundary (marked in red) at the grid points and (b) nonuniform Cartesian grid scheme by scaling the spatial step for $\hat{y}$ by $\sqrt{3}$ with respect to $\Delta x$, which enables the periodic boundary completely sampled at the grid points (marked as blue points).

and $\exp (-i 2 \pi \xi / 3)$ for the $f_{2}^{\prime}-f_{2}^{\prime \prime}$ and $f_{1}^{\prime}-f_{1}^{\prime \prime}$ periodic boundaries, respectively. Another choice of propagation constant for the $\Gamma-M$ direction is $[(\pi / a) \hat{x}]+[(\pi / a \sqrt{3}) \hat{y}]$, which is also shown in Fig. 1(b). The phase constants for both periodic boundaries will accordingly change. In theory, the photonic band diagrams are identical, regardless of the choice of propagation constant as long as they are translational invariant with respect to the reciprocal lattice vectors. However, the numerical results suffer a small difference due to a slight circular asymmetry. Circles formed in an $x-y$ grid do not possess an exact $60^{\circ}$ rotational symmetry. Even so, it is found [15] that the margin of error is well below $1 \%$ when an order- $N$ method [16] is applied.

As shown in Fig. 3(a), the rhombus unit cell forms a $60^{\circ} / 120^{\circ}$ angle between neighboring boundaries. If it were to be directly mapped to a uniform isotropic Cartesian grid, two of its periodic boundaries $f_{1}^{\prime}-f_{1}^{\prime \prime}$ cannot be completely sampled at the grid points. This is because the desired boundary conditions cannot be directly enforced on the physical boundary but, rather, on an auxiliary boundary, which is a staircased approximation of the physical boundary. In general, those values that are not directly given at the grid points can only be calculated from their nearest neighbors. Unfortunately, this leads to instability because the electromagnetic field at the boundary carries important phase information. A slight error in the phase will cause the calculation to be extremely unstable. As isotropic grid on a Cartesian coordinate only natively describes a $45^{\circ}$ straight line; a $60^{\circ}$ line would require a scaling of axes by $\sqrt{3}$, as schematically shown in Fig. 3(b).

Increasing the spatial step $\Delta y$ for the $\hat{y}$-axis also increases the numerical dispersion error [10]. When the numerical accuracy is a concern for long iterations, it is more desirable to reduce $\Delta x$ by a factor of $\sqrt{3}$. The time step $\Delta t$ will be accordingly adjusted to ensure that the FDTD simulation still satisfies the stability condition given by the Courant constant [10]. It is clear that for any general nonorthogonal 2-D unit cell, $\Delta x$ and $\Delta y$ can always be scaled so that the parallelogram unit cell will be precisely mapped onto the Cartesian FDTD grid.

It is worth noting that the triangular lattice photonic crystals can also be periodically extended with the rectangle as the unit cell [17] (marked with dotted lines in Fig. 2). It is straightforward to implement a rectangle unit cell in the Cartesian FDTD method. However, as the rectangular lattice has a primitive lattice of $\boldsymbol{a}_{1}$ and $\boldsymbol{a}_{2}-\boldsymbol{a}_{1} / 2$ and two air holes per unit cell, the photonic band structure represents a "folded version" of a real band diagram - one which would have "extra" bands that would need to be identified and unfolded. One way to overcome this unit-cell problem with the standard FDTD is to place a dipole source in the same spot of every copy of the primitive cell represented within the larger rectangular grid that is being simulated and to then adjust the phase of the waveform that is applied to satisfy each particular propagation constant $\boldsymbol{k}$ [7]. This can be adapted to the simple one-time-step "ping" by having the second impulse, at point $\boldsymbol{r}^{\prime}$ relative to the first, appear on both layers of the FDTD grid, weighted by $\cos \left(\boldsymbol{k} \cdot \boldsymbol{r}^{\prime}\right)$ and $\sin \left(\boldsymbol{k} \cdot \boldsymbol{r}^{\prime}\right)$, respectively.

However, not all 2-D periodic structures can be described with a rectangular unit cell. As more primitive unit cells are included in the rectangular unit cell for the purpose of calculation, more bands will be folded into the reduced Brillouin zone, which becomes increasingly difficult to unfold. In contrast, our choice of parallelogram unit cell and scaled spatial step can handle general 2-D photonic crystals on a Cartesian FDTD grid scheme.

\section{B. Split-Field Formulation}

Fig. 4 shows the grid scheme for applying the general periodic boundary condition. For the sake of clarity, the algorithm will be explained for the electromagnetic field in a 2-D case. The situation with the full 3-D case can be similarly applied. In the 2-D case, Maxwell's equations are simplified into two 


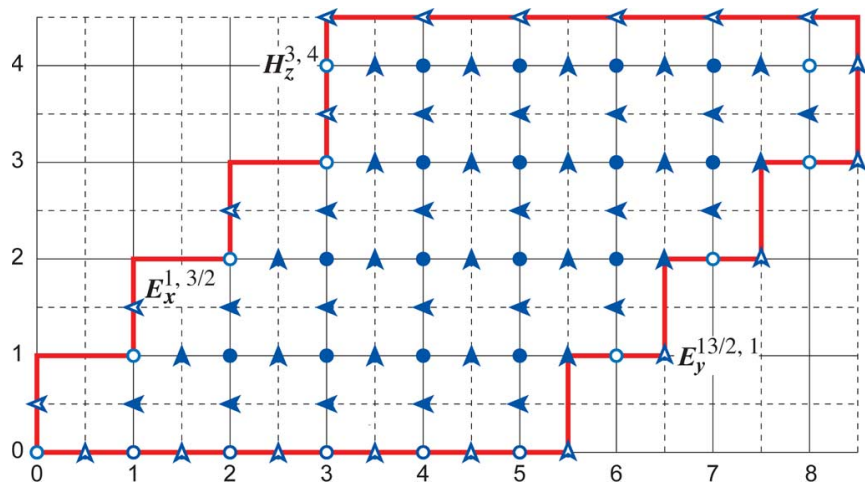

Fig. 4. Noncollocated grid scheme for the FDTD method. The bold line delineates the boundary for the nonorthogonal unit cell. The $E_{x}, E_{y}$, and $H_{z}$ components are sampled and shown as the left arrows, up arrows, and circles, respectively, in the figure. The open symbols indicate that those elements are to be calculated by the periodic boundary condition.

independent sets of equations whose solutions are classified as transverse electric $\left(T E_{z}\right)$ modes, i.e.,

$$
\begin{aligned}
\frac{\partial H_{z}(\boldsymbol{\rho})}{\partial y} & =\frac{\partial \varepsilon(\boldsymbol{\rho}) E_{x}(\boldsymbol{\rho})}{\partial t} \\
\frac{\partial H_{z}(\boldsymbol{\rho})}{\partial x} & =-\frac{\partial \varepsilon(\boldsymbol{\rho}) E_{y}(\boldsymbol{\rho})}{\partial t} \\
\frac{\partial E_{y}(\boldsymbol{\rho})}{\partial x}-\frac{\partial E_{x}(\boldsymbol{\rho})}{\partial y} & =-\frac{\partial \mu_{0}(\boldsymbol{\rho}) H_{z}(\boldsymbol{\rho})}{\partial t}
\end{aligned}
$$

and transverse magnetic $T M_{z}$ waves. For $T E_{z}$ waves, there exist three nonzero $E_{x}, E_{y}$, and $H_{z}$ field components. The locations of the $E_{x}, E_{y}$, and $H_{z}$ components are shown in Fig. 4 as left arrows, up arrows, and circles, respectively.

The grid scheme is developed from the original Yee's interlaced space lattice. It is extended by half a unit cell so that all electromagnetic field components will border the domain boundary for all three directions. This extension leads to an equal dimension of electromagnetic field components. When the algorithm is implemented in the parallel environment, this modified grid scheme has an added benefit that the synchronization between processors is balanced between $\boldsymbol{E}$ and $\boldsymbol{H}$ components.

The bold line in Fig. 4 delineates the parallelogram unit cell that is represented in the Cartesian FDTD grid, assuming it has a dimension of $5 \times 4$. Using the central difference approximation, the explicit time-stepping relations can be derived from (5)-(7). $E_{x, n+1 / 2}^{i, j+1 / 2}$ is a shorthand expression for $E_{x}(i \Delta x,(j+1 / 2) \Delta y,(n+1 / 2) \Delta t)$, i.e.,

$$
\begin{aligned}
E_{x, n+1 / 2}^{i, j+1 / 2}= & \frac{1-\frac{\sigma^{i, j+1 / 2} \Delta t}{2 \varepsilon^{i, j+1 / 2}}}{1+\frac{\sigma^{i, j+1 / 2} \Delta t}{2 \varepsilon^{i, j+1 / 2}}} E_{x, n-1 / 2}^{i, j+1 / 2} \\
& +\frac{1-\frac{\Delta t}{2 \varepsilon^{i, j+1 / 2}}}{1+\frac{\sigma^{i, j+1 / 2} \Delta t}{2 \varepsilon^{i, j+1 / 2}}}\left(\frac{H_{z, n}^{i, j+1}-H_{z, n}^{i, j}}{\Delta y}\right) \\
E_{y, n+1 / 2}^{i+1 / 2, j+1}= & \frac{1-\frac{\sigma^{i+1 / 2, j+1} \Delta t}{2 \varepsilon^{i+1 / 2, j+1}}}{1+\frac{\sigma^{i+1 / 2, j+1} \Delta t}{2 \varepsilon^{i+1 / 2, j+1}}} E_{y, n-1 / 2}^{i+1 / 2, j+1} \\
& +\frac{1-\frac{\Delta t}{2 \varepsilon^{i+1 / 2, j+1}}}{1+\frac{\sigma^{i+1 / 2, j+1} \Delta t}{2 \varepsilon^{i+1 / 2, j+1}}}\left(\frac{H_{z, n}^{i, j+1}-H_{z, n}^{i+1, j+1}}{\Delta x}\right)
\end{aligned}
$$

$$
\begin{aligned}
H_{z, n+1}^{i, j+1}= & \frac{1-\frac{\sigma_{m}^{i, j+1} \Delta t}{2 \mu^{i, j+1}}}{1+\frac{\sigma_{m}^{i, j+1} \Delta t}{2 \mu^{i, j+1}}} H_{z, n}^{i, j+1} \\
& +\frac{1-\frac{\Delta t}{2 \mu^{i, j+1}}}{1+\frac{\Delta t}{2 \mu^{i, j+1}}}\left(\begin{array}{c}
\frac{E_{x, n+1 / 2}^{i, j+3 / 2}-E_{x, n+1 / 2}^{i, j+1 / 2}}{\Delta y} \\
+\frac{E_{y, n+1 / 2}^{i-1 / 2, j+1}-E_{y, n+1 / 2}^{i+1 / 2, j+1}}{\Delta x}
\end{array}\right) .
\end{aligned}
$$

From (8)-(10), it shows that some field elements in the unit cell cannot be calculated with the central difference equations because they require the knowledge of field values outside the unit cell. They are identified as open symbols in Fig. 4. These elements need to be calculated by the periodic boundary condition. However, as can be seen in Fig. 4, the field component $H_{z}$ at both the left-hand side and the right-hand side of the unit cell are unknown. This is because, according to (10), calculating $H_{z}$ requires known $E_{y}$ values to its left and right, as well as $E_{x}$ values to its top and bottom. As the jagged left-right boundary put $H_{z}$ as the last component on both the left and the bottom of the simulation domain, none of these boundary values can be determined by (10).

To resolve this dilemma, we employ a split-field formulation of Maxwell's equations, where the magnetic field component $H_{z}$ is split into two orthogonal components $H_{z x}$ and $H_{z y}$. The differential equations are then given by

$$
\begin{aligned}
H_{z x, n+1}^{i, j+1}= & \frac{1-\frac{\sigma_{m}^{i, j+1} \Delta t}{2 \mu^{i, j+1}}}{1+\frac{\sigma_{m}^{i, j+1} \Delta t}{2 \mu^{i, j+1}}} H_{z x, n}^{i, j+1} \\
& +\frac{1-\frac{\Delta t}{2 \mu^{i, j+1}}}{1+\frac{\Delta t}{2 \mu^{i, j+1}}}\left(\frac{E_{y, n+1 / 2}^{i-1 / 2, j+1}-E_{y, n+1 / 2}^{i+1 / 2, j+1}}{\Delta x}\right) \\
H_{z y, n+1}^{i, j+1}= & \frac{1-\frac{\sigma_{m}^{i, j+1} \Delta t}{2 \mu^{i, j+1}}}{1+\frac{\sigma_{m}^{i, j+1} \Delta t}{2 \mu^{i, j+1}}} H_{z y, n}^{i, j+1} \\
& +\frac{1-\frac{\Delta t}{2 \mu^{i, j+1}}}{1+\frac{\Delta t}{2 \mu^{i, j+1}}}\left(\frac{E_{x, n+1 / 2}^{i, j+3 / 2}-E_{x, n+1 / 2}^{i, j+1 / 2}}{\Delta y}\right) .
\end{aligned}
$$

By splitting the $H_{z}$ field, $H_{z x}$ is only dependent on $E_{y}$, and therefore, its value on the right boundary can be calculated. The same is true for $H_{z y}$ on the left-hand-side boundary. As a result, the Bloch condition is then given by

$$
\begin{aligned}
H_{z x}^{j, j} & =H_{z x}^{j+a, j} \exp (i \boldsymbol{k} \cdot a \hat{x}) \\
H_{z y}^{j+a, j} & =H_{z y}^{j, j} \exp (-i \boldsymbol{k} \cdot a \hat{x})
\end{aligned}
$$

in which $a$ is the length of the unit cell in the $\hat{x}$ direction, and $\boldsymbol{k}$ is the wave vector that is normalized by the unit cell. For the unit cell shown in Fig. 4, $a=5$. 


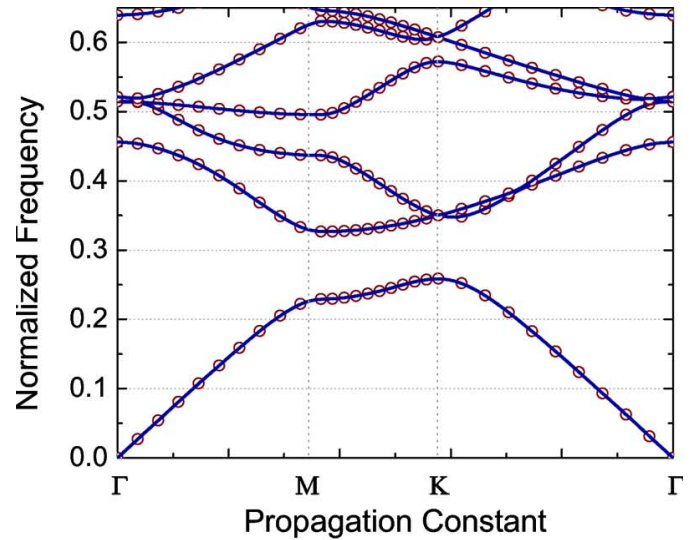

(a)

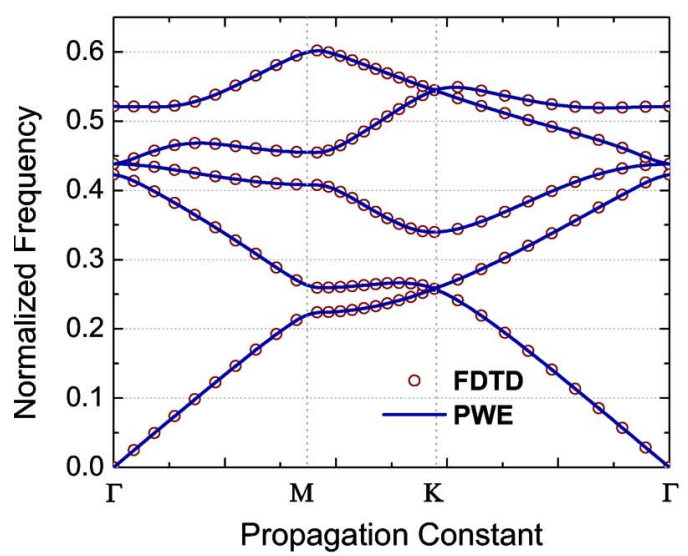

(b)

Fig. 5. Photonic band diagram of (a) the $T E_{z}$ and (b) the $T M_{z}$ modes for 2-D triangular lattice photonic crystals using the 2-D FDTD method and a 2-D PWE method. The dielectric slab has an effective index of 2.76 and a triangular lattice of air holes $r / a$ of 0.3 .

\section{Triangular LAtTiCe Photonic CRYstals}

In Section II, we have described the FDTD algorithm for calculating the dispersion relations of photonic crystals with a nonorthogonal unit cell. In this section, the numerical method will be applied for a 2-D triangular lattice photonic crystal slab. The dielectric slab, which is assumed to be suspended in the air, has a refractive index $n$ of 3.4 and a normalized thickness $d / a$ of 0.6. Air holes with a normalized radius $r / a$ of 0.3 perforate the dielectric membrane. The calculation will be performed with both 2-D and 3-D FDTD methods. For 2-D calculations, the dielectric constant of the slab is approximated by the effective index for the zeroth-order mode that is confined in the dielectric slab. The dispersion relation will be compared with the 2-D PWE method using the same effective index. We also perform a fully 3-D FDTD calculation with a PML ABC [18]-[20] that terminates the vertical $\hat{z}$ direction. The result will be compared with the 3-D FEM. Note that the choice of triangular lattice for the comparison has simply been because of the wealth of existing results. However, as discussed earlier, the method can be applied to any general 2-D lattice.

Fig. 5 shows the photonic band diagram of the $T E_{z}$ and $T M_{z}$ modes for 2-D triangular lattice photonic crystals. It plots the frequency-versus-propagation-constant relations for the modes along the high-symmetry directions $\Gamma-M, M-K$, and $K-\Gamma$

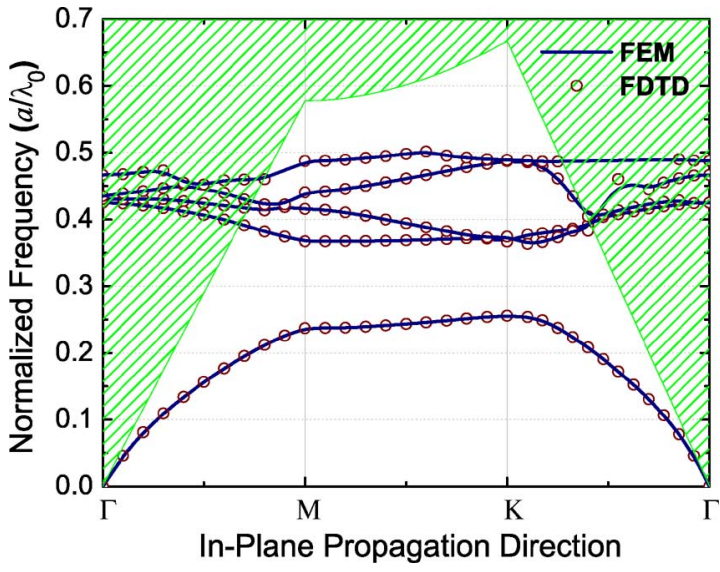

Fig. 6. Photonic band structure for a suspended membrane photonic crystals, which is calculated with the 3-D FDTD method. The dielectric membrane has a refractive index of 3.4 and normalized thickness of 0.6 . Triangular lattice air holes that perforate the membrane have a normalized radius of 0.3 .

of the lattice. Those high-symmetry locations are defined in Fig. 1. The triangular lattice creates a bandgap for in-plane $T E_{z}$ modes. In the calculation, the dielectric assumes a refractive index of 2.76 given by the effective index of the fundamental $T E$ mode for an infinite slab, whose refractive index is 3.4 , and the normalized thickness is 0.6. The FDTD and PWE results are shown in Fig. 5 as the open circles and the solid lines, respectively. The first bandgap of the $T E_{z}$ mode is formed by the first and second bands; however, an in-plane bandgap for the $T M_{z}$ modes does not exist. The difference in normalized frequencies from the two approaches is less than $1 \%$.

We also extend the algorithm into the 3-D FDTD and calculate the photonic band structure for the even modes (TE like) in a triangular lattice photonic crystal membrane. Fig. 6 shows the 3-D FDTD result as open circles as well as the FEM results as solid lines for comparison. The shaded area is the light cone of the air cladding that is mapped on the respective propagation directions. The existence of a high-index substrate will allow the field to evanescently couple to the radiation modes of the substrate, which leads to a significant power loss. The photonic crystal modes in the shaded region are not strictly guided by the dielectric slab. Hence, the calculation of frequencies for those modes is less accurate with the FDTD, as they have a relatively short lifetime and a broad spectral response. The agreement between the two numerical approaches can be clearly identified in the figure, particularly for the below-light-line region where the difference is less than $2 \%$.

Comparing the results from the 3-D FDTD and the effective index 2-D FDTD, as shown in Fig. 5, the first band shares a strong semblance. However, the discrepancy becomes more drastic for higher bands. That is because higher transverse modes are less localized in plane and more localized in the slab vertically. Hence, 2-D effective index underestimates the vertical confinement and leads to a higher eigenfrequency. Additionally, the $z$ dependence of photonic crystal modes is also a function of the propagation constant. As a result, the discrepancy in the dispersion relation from a full 3-D calculation and 2-D effective index approximation is also propagation constant dependent. Because of this, the group velocity and dispersion properties of a photonic crystal mode, which is given 
by the higher order derivative of the band, can be significantly different from that of the 2-D result. It is an important issue in such applications as superprisms, where the higher order dispersion relation of the photonic crystals is the key to the device design.

Additional band structures for triangular lattice photonic crystals on oxides and high-index substrates can be found in [21] and [22]. The flexibility of the FDTD method is that a general epitaxial structure can be easily incorporated. As a final comment, this Cartesian FDTD method can also be similarly extended to calculate the band structure for 3-D photonic crystals.

\section{Discussion AND CONCLUSION}

In summary, a fully 3-D FDTD method is presented for calculating the dispersion relation of 2-D photonic crystals. The choice of a parallelogram primitive cell and split-field formulation of Maxwell's equation enables the Cartesian FDTD to handle the general 2-D space lattice. The photonic band diagram for the 2-D TE/TM modes in triangular lattice photonic crystals that is calculated by the 2-D FDTD method is in excellent agreement with the results from the PWE method. We also show that the fully 3-D FDTD calculation of the triangular lattice photonic crystal in a dielectric slab agrees well with the 3-D FEM solution. However, being a fully explicit computation, the FDTD is more desirable for a calculation that requires a large simulation domain because the computation time $o(N)$ is linearly proportional to the mesh density. A comparison of the 2-D and 3-D band diagrams indicates that the 2-D calculation using an effective index approximation can result in a considerable error for higher order modes. It is an important issue in such applications as superprisms, where the group velocity dispersion of the photonic crystals is the key to the device design.

\section{ACKNOWLEDGMENT}

Computation for this work was supported in part by the University of Southern California Center for High-Performance Computing and Communications.

\section{REFERENCES}

[1] E. Yablonovitch, "Inhibited spontaneous emission in solid-state physics and electronics," Phys. Rev. Lett., vol. 58, no. 20, pp. 2059-2062, May 1987.

[2] S. John, "Strong localization of photons in certain disordered dielectric superlattices," Phys. Rev. Lett., vol. 58, no. 23, pp. 2486-2489, Jun. 1987.

[3] J. D. Joannopoulos, R. D. Meade, and J. N. Winn, Photonic Crystals: Molding the Flow of Light. Princeton, NJ: Princeton Univ. Press, 1995.

[4] W. Kuang, J. R. Cao, S.-J. Choi, J. D. O’Brien, and P. D. Dapkus, "Classification of modes in suspended membrane 19-missing-hole photonic crystal microcavities," J. Opt. Soc. Amer. B, Opt. Phys., vol. 22, no. 5, pp. 1092-1099, May 2005.

[5] C. Oubre and P. Nordlander, "Finite-difference time-domain studies of the optical properties of nanoshell dimers," J. Phys. Chem. B, vol. 109, no. 20, pp. $10042-10051,2005$.

[6] W. J. Kim, "Full vectorial finite element analysis of photonic crystal devices: Application to low-loss modulator," Ph.D. dissertation, Univ. Southern California, Los Angeles, CA, 2004

[7] S. Fan, P. R. Villeneuve, and J. D. Joannopoulos, "Large omnidirectional band gaps in metallodielectric photonic crystals," Phys. Rev. B, Condens. Matter, vol. 54, no. 16, pp. 11 245-11251, Oct. 1996.
[8] J.-F. Lee, R. Palendech, and R. Mittra, "Modeling three-dimensional discontinuities in waveguides using nonorthogonal FDTD algorithm," IEEE Trans. Microw. Theory Tech., vol. 40, no. 2, pp. 346-352, Feb. 1992.

[9] K. S. Yee, "Numerical solution of initial boundary value problems involving Maxwell's equation in isotropic media," IEEE Trans. Antennas Propag., vol. AP-14, no. 3, pp. 302-307, May 1966.

[10] A. Taflove and S. C. Hagness, Computational Electrodynamics: The Finite-Difference Time-Domain Method. Norwood, MA: Artech House, 2000.

[11] R. D. Meade, A. M. Rappe, K. D. Brommer, and J. D. Joannopoulos, "Accurate theoretical analysis of photonic band-gap materials," Phys. Rev. B, Condens. Matter, vol. 48, no. 11, pp. 8434-8437, Sep. 1993.

[12] K. Sakoda, Optical Properties of Photonic Crystals. New York: Springer-Verlag, 2001.

[13] M. Plihal and A. A. Maradudin, "Photonic band structure of twodimensional systems: The triangular lattice," Phys. Rev. B, Condens. Matter, vol. 44, no. 16, pp. 8565-8571, Oct. 1991.

[14] P. R. Villeneuve and M. Piche, "Photonic band gaps in two-dimensional square and hexagonal lattices," Phys. Rev. B, Condens. Matter, vol. 46, no. 8, pp. 4969-4972, Aug. 1992.

[15] W. Kuang, "Photonic crystal waveguides," Ph.D. dissertation, Univ. Southern California, Los Angeles, CA, 2005.

[16] C. Chan, Q. Yu, and K. Ho, "Order-n spectral method for electromagneticwaves," Phys. Rev. B, Condens. Matter, vol. 51, no. 23, pp. 16 635-16642, Jun. 1995.

[17] A. Chutinan and S. Noda, "Waveguides and waveguide bends in twodimensional photonic crystal slabs," Phys. Rev. B, Condens. Matter, vol. 62, no. 7, pp. 4488-4492, Aug. 2000.

[18] J.-P. Berenger, "A perfectly matched layer for the absorption of electromagnetic waves," J. Comput. Phys., vol. 114, no. 2, pp. 185-200, 1994.

[19] J.-P. Berenger, "Perfectly matched layer for the FDTD solution of wave-structure interaction problems," IEEE Trans. Antennas Propag., vol. 44, no. 1, pp. 110-117, Jan. 1996.

[20] J.-P. Berenger, "Three-dimensional perfectly matched layer for the absorption of electromagnetic waves," J. Comput. Phys., vol. 127, no. 2, pp. 363-379, 1996

[21] W. Kuang, C. Kim, A. Stapleton, W. J. Kim, and J. D. O'Brien, "Calculated out-of-plane transmission loss for photonic-crystal slab waveguides," Opt. Lett., vol. 28, no. 19, pp. 1781-1783, Oct. 2003.

[22] W. Kuang, W. J. Kim, A. Mock, and J. D. O'Brien, "Propagation loss of line-defect photonic crystal slab waveguides," IEEE J. Sel. Topics Quantum Electron., vol. 12, no. 6, pp. 1183-1195, Nov./Dec. 2006.

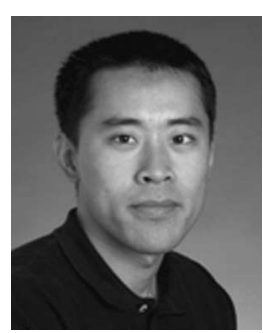

Wan Kuang (S'02-M'06) received the B.S.E.E. and M.S.E.E. degrees from Chongqing University of Posts and Telecommunications, Chongqing, China, and the $\mathrm{Ph} . \mathrm{D}$. degree from the University of Southern California, Los Angeles, all in electrical engineering.

$\mathrm{He}$ is currently an Assistant Professor with the Department of Electrical and Computer Engineering, Boise State University, Boise, ID. His research interests include numerical methods in electromagnetics and photonic bandgap material.

Woo Jun Kim received the Ph.D. degree in electrical engineering from the University of Southern California, Los Angeles. He is currently with Samsung Electronics, Seoul, South Korea.

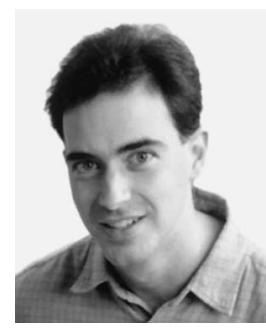

John D. O'Brien (M'98-SM'02) received the B.S. degree in electrical engineering from Iowa State University, Ames, in 1991 and the M.S. and Ph.D. degrees in applied physics from the California Institute of Technology, Pasadena, in 1993 and 1996, respectively.

In 1997, he was an Assistant Professor with the University of South Carolina, Columbia, where he is currently a Professor of electrical engineeringelectrophysics. His research interests include nanophotonics and photonic crystal devices.

Dr. O'Brien was the recipient of the Presidential Early Career Award for Scientists and Engineers in 1999 and the NSF Career Award in 2000. He is currently an Associate Editor of IEEE TRANSACTIONS ON NANOTECHNOLOGY. 